Revue de l'Institut des langues et cultures

d'Europe, Amérique, Afrique, Asie et Australie

13 | 2010

Les voies incertaines de la démocratisation

\title{
Documents pour l'Histoire. Regards pour une nouvelle démocratie au Chili
}

Documents for History. A view on a new democracy in Chili

\section{Olga Lobo}

\section{OpenEdition}

\section{Journals}

Édition électronique

URL : http://journals.openedition.org/ilcea/911

DOI : 10.4000/ilcea.911

ISSN : 2101-0609

\section{Éditeur}

UGA Éditions/Université Grenoble Alpes

\section{Édition imprimée}

ISBN : 978-2-84310-181-6

ISSN : $1639-6073$

Référence électronique

Olga Lobo, « Documents pour l'Histoire. Regards pour une nouvelle démocratie au Chili », ILCEA [En ligne], 13 | 2010, mis en ligne le 30 novembre 2010, consulté le 02 mai 2019. URL : http:// journals.openedition.org/ilcea/911 ; DOI : 10.4000/ilcea.911

Ce document a été généré automatiquement le 2 mai 2019.

(c) ILCEA 


\section{Documents pour l'Histoire. Regards pour une nouvelle démocratie au Chili}

Documents for History. A view on a new democracy in Chili

\section{Olga Lobo}

Una sociedad necesita mirarse en los espejos del arte

para comprenderse.

Fernando BIRRI

1 Le résultat du plébiscite du 5 octobre 1988 , où $55,99 \%$ des Chiliens refusèrent la continuation de Pinochet au pouvoir, exprimait la volonté populaire d'un retour à la voie démocratique qui deviendra effectif lors de l'élection à la présidence de Patricio Aylwin le 14 décembre 1989.

2 Bien que, en effet, la victoire du «non » dans le plébiscite ait signifié un point d'inflexion dans le développement historico-politique du Chili, la voie empruntée s'est rapidement avérée incertaine dans la mesure où de nombreuses difficultés ont fait obstacle à la consolidation d'une démocratie "sans ambages". Ainsi, des qualifications telles que " démocratie surveillée » (democracia vigilada) ou " démocratie sous la tutelle de l'armée » (democracia tutelada) ont défini pendant longtemps les limites d'une démocratie qui devait faire face à de nombreuses adversités.

3 En effet, les difficultés pour passer d'une économie (ultra)libérale à une économie de marché plus soucieuse de la protection sociale, les obstacles pour abolir et remplacer aussi bien la constitution de $1980^{1}$ que, entre autres ${ }^{2}$, la loi électorale de $1978^{3}$ ou encore le combat pour récupérer un véritable État de droit ${ }^{4}$, sont quelques-uns des facteurs qui nous permettent de nous interroger sur l'«authenticité » d'une démocratie qui nonobstant semble stable, consolidée.

4 Cette démocratie, qui a donc avancé péniblement sous les verrous militaires, s'est établie, depuis ses débuts, sur la base d'un discours et d'une pratique politique de consensus. Au moyen du consensus, les instances politiques ont cherché à combattre la menace 
permanente d'une involution offrant au peuple chilien une démocratie qui pourrait être définie, tel que l'explique Nelly Richard (2000, p. 175), comme une démocratie du possible, basée davantage sur la transaction, grâceaux techniques de la négociation, que sur une véritable prise de décisions libre et responsable. Une démocratie, donc, placée sous le signe de la continuité ${ }^{5}$ et qui se veut « neutre », prudente...

Dix ans après le plébiscite, la détention de Pinochet à Londres allait cependant bouleverser la marche de cette démocratie consensuelle au Chili. Au-delà de tout ce que l'arrestation de Pinochet à Londres signifie pour le droit international ${ }^{6}$, les conséquences sociales et politiques se révèlent d'une importance capitale pour le Chili.

6 L'affaire Pinochet ouvre, en effet, une nouvelle étape dans la «transition à la démocratie » aussi bien au niveau des sphères du pouvoir (pour qui Pinochet commençait à représenter un fardeau trop lourd), qu'au niveau de « la rue ».

7 L'affaire Pinochet montra que les blessures du passé étaient bel et bien ouvertes mais, en même temps, les Chiliens ont rompu la loi du silence imposée par la stratégie d'oubli et d'impunité des militaires.

L'affaire Pinochet - explique Nelly Richard (2000, p. 177) - fit voler en éclat cette zone d'accumulation du non-dit où se cachaient en silence les frustrations de la Transition. Un spectaculaire «retour du refoulé » fit sauter tout l'appareil rhétorique de la Transition (mesure, calcul, modération, etc.) qui avait bloqué le travail de la mémoire au nom d'une prudence faite d'invisibles stratégies d'occultation du conflit [...] les réactions qui se déchaînèrent dans la rue à propos de l'affaire Pinochet ont démontré le caractère artificiel du consensus [...]. L'affaire Pinochet produisit un dérangement symbolique à l'intérieur de la marche politique consensuelle qui paraissait si bien rodée, et démontra aussi la disjonction existante entre le discours politique et les pratiques sociales...

8 En effet, comme le dénonçaient depuis un "espace de l'arrière-garde » les associations internationales ou locales (le projet « droits de l'homme $»^{7}$, la Vicaria de la solidaridad ${ }^{8}$, le CODEPU $^{9}$, la FASIC $^{10}$ ), l'aspect le plus déterminant dans la fragilisation de la jeune démocratie chilienne était l'absence d'un véritable travail de la mémoire, longtemps rejeté dans la logique du discours et des pratiques politiques du consensus.

Certes, les quelques efforts timides qui, depuis le début de la démocratie en 1990 et jusqu'à la détention de Pinochet à Londres, avaient été mis en œuvre afin d'établir la vérité ${ }^{11}$, rendent compte d'une certaine volonté de lutte contre la stratégie d'oubli et de terreur qu'avaient voulu imposer les militaires. Or ces actions relèvent d'une pratique de la justice dite justice transitionnelle (Kritz, 1995), qui ne se contente pas des instruments du droit pénal et qui est loin de satisfaire les besoins et les exigences des victimes et d'autres acteurs sociaux.

10 La disjonction dont parlait Richard plus haut s'expliquerait donc par la frustration qu'avait provoquée cette pratique de justice transitionnelle dans la mesure où les victimes avaient considéré les commissions de vérité « comme une simple première étape vers la condamnation des coupables » (Lefranc, 2008, p. 68).

11 À l'occasion de l'affaire Pinochet on a pu assister ainsi à l'éclosion des manifestations contre une justice jusqu'alors très... injuste: d'une part parce qu'elle imposait la réconciliation par décret et interdisait tout exercice de la mémoire (Lefranc, 2002, p. 133), et, d'autre part, dans la mesure où, grâce aux techniques de la sociologie, elle réduisait les victimes à la qualité de chiffres et l'Histoire à des faits sans conséquence, selon le 
« régime plat et neutre du quantitatif et du statistique, du numéraire et du sériel » (Richard, 2000, p. 181).

12 L'arrestation de Pinochet ainsi que la pression internationale ont permis à une grande partie de la société chilienne de manifester publiquement le besoin d'une rupture plus claire, plus radicale, vis-à-vis du passé. L'éclosion du discours consensuel a également permis la mise en cause du discours officiel d'une mémoire institutionnelle, créant ainsi un interstice où une autre mémoire est possible, où une autre justice est possible. En provoquant une crise dans le consensus, «la rue » a réussi à déstabiliser, créer le débat, contester le discours hégémonique. Désormais plus de silence, plus de consensus non plus. L'artificialité de cette société, soi-disant, pacifiée à coups de décrets éclatait en même temps que « la rue » retrouvait un rôle central de revendication de justice.

À mesure que le procès judiciaire évoluait dans le sens que les associations des victimes et une bonne partie de la société chilienne (et mondiale) le souhaitaient, à mesure aussi que les gens, notamment les exilés chiliens en Europe, gagnaient en force morale et en espoir, les partisans de Pinochet ont senti, eux aussi, le besoin de montrer au monde qu'ils étaient là, nombreux, et prêts à tout pour défendre la figure d'un homme qu'ils considèrent comme l'un des pères de la patrie. La rue devint ainsi la scène visible de la confrontation directe de ces « deux Chili ».

Le sentiment d'incompréhension face à l'exhibition d'un soutien quelque peu grotesque à Pinochet (allant jusqu'à la confection de T-shirts avec l'inscription «I love Pinochet » et d'autres "produits dérivés») de la part de ses partisans a d'ailleurs été l'objet du film documentaire intitulé I love Pinochet, opera prima cinématographique de la réalisatrice chilienne Marcela Said qui, au moyen d'un récit à la première personne, prend la parole pour s'interroger sur les raisons d'un tel soutien.

D'un point de vue très différent Patricio Guzmán consacre également son film Le cas Pinochet à cette affaire judiciaire qui allait bouleverser la marche sociopolitique du Chili démocratique.

Ce sont ces deux films que l'on s'efforcera d'analyser ici afin d'étudier leur rôle en tant que documents pour l'Histoire et d'expliquer dans quelle mesure ils apportent à la lecture $\mathrm{du}$ fait historique qu'ils analysent un regard critique qui se constitue en véritable revendication d'une nouvelle démocratie pour leur pays.

\section{Cinéma documentaire chilien : regards sur et pour l'Histoire}

Malgré les difficultés pour s'introduire dans l'« espace public »12, le cinéma documentaire chilien vit un certain essor depuis l'avènement de la démocratie au Chili ${ }^{13}$.

À la suite du coup d'État en 1973 et jusqu'à nos jours, un grand nombre de réalisateurs ont consacré leur travail à la création d'un langage où esthétique et éthique se rejoignent dans une même poétique de résistance ${ }^{14}$. Ce sont des créations qui, au-delà de leur qualité artistique, se constituent en véritables espaces de mémoire, cherchant à établir un dialogue permanent avec l'Histoire pour mieux la questionner. Tel est le cas de deux documentaires qui font l'objet de notre étude: El caso Pinochet (Le cas Pinochet) de Patricio Guzmán (1941) et I love Pinochet de Marcela Said (1972), les deux apparus en 2001. 

l'importance que l'arrestation de Pinochet aura dans l'action de la justice et la restitution de la mémoire au Chili après 1999. La première séquence du film se situe quelque temps après le retour de Pinochet au Chili, dans le désert (au nord du Chili) où la famille d'un groupe de disparus se rend, à la demande du juge Juan Guzmán, afin d'y trouver leurs corps. Pendant ces premières minutes du film, Guzmán semble indiquer déjà la position que son documentaire va prendre en tant qu'espace de parole pour les victimes : ce sont les victimes et non pas le bourreau (malgré la place centrale qu'il occupe dans le titre du film) qui se révèlent être les véritables protagonistes du film.

détention de Pinochet. Ce sont eux qui auront l'occasion de raconter en détail les charges contre le dictateur, à défaut (et en attendant) de pouvoir le faire devant la justice, ce sont eux qui porteront, finalement, un message d'espoir et d'appel à l'action contre l'impunité et contre l'oubli. victimes, les plans serrés, les silences, les regards profonds et directs qui occupent l'image... créent un véritable espace d'intimité entre les deux sujets qui se trouvent des deux côtés de la caméra. En effet, si les images des séquences les plus "prosaïques" illustrent le récit des faits historiques frôlant le reportage journalistique, où se succèdent récit en off et entretiens avec des experts, le documentaire alterne aussi ces séquences avec de longs plans des victimes racontant leur expérience. Leur récit de la mémoire s'interrompt, comme on le disait plus haut, par de longs silences, des hésitations et des blancs donnant lieu à d'émouvants moments de réflexion, aussi, pour le spectateur, invitant, comme on le verra, à une mémoire qui cherche à construire un futur démocratique plus juste et, dès lors, plus « authentique ». 
Au moyen de son utilisation de l'image et du récit, El caso Pinochet deviendra, grâce à cette connivence entre esthétique et éthique, cet espace (à la fois inexistant et recherché, dès lors utopique) où les victimes de la dictature peuvent enfin s'exprimer, dénoncer, raconter ; le documentaire se dévoile ainsi, disions-nous (Lobo, 2006, p. 205), un espace du possible où le réalisateur cède la parole, il fait et laisse parler, il fait et laisse regarder, articulant dans son discours un hommage aux victimes grâce au pathos de la mémoire de ces gens qui expriment (laissent couler) face à la caméra, complice de leur souffrance, les souvenirs qui les hantent.

En mettant en exergue le travail de mémoire des familles dès les premières séquences du film, Guzmán semble en effet souligner l'importance que l'arrestation de Pinochet aura pour la suite des événements au Chili : « chaque corps retrouvé, affirme le juge Guzmán dans ces premiers minutes du documentaire, implique un pas de plus vers la paix sociale ».

le ton de frustration vis-à-vis de la libération de Pinochet, le film interprète son arrestation comme une des circonstances déterminantes dans un début indéniable de changement des mentalités (et des attitudes) tant au niveau social que politique. Il insiste également sur la valeur symbolique de cette détention dans la mesure où elle représente une victoire pour ces voix qui avaient été longtemps maintenues à l'écart, sous le silence et l'oubli.

Cette analyse est développée à maintes reprises dans le film aussi bien dans les entretiens des experts que dans le récit en off: ainsi, l'action de la justice espagnole est interprétée comme la continuation et le complément du travail mené par les Associations d'aide aux victimes (entre autres la Vicaría de la solidaridad) et les avocats chiliens (tels Garretón ou Joan Garcés) depuis 1973.

30 La démarche espagnole, affirme le réalisateur, a permis, d'une part, que ce travail puisse avoir un sens en se concrétisant dans une action de justice réelle ; elle a servi, d'autre part, de détonateur pour que les victimes osent dénoncer et, enfin, pour que Juan Guzmán ait pu continuer le procès contre le dictateur au Chili jusqu'à son arrestation :

Pendant le séjour de Pinochet à Londres - explique la voix off dans la partie finale $\mathrm{du}$ documentaire (1 h $34 \mathrm{~min} 35 \mathrm{~s})$ - le juge Juan Guzmán reçoit les plaintes chiliennes, les familles des victimes présentent plus de deux cents requêtes contre Pinochet, dénonçant la loi d'amnistie. La population n'a plus peur et la justice rattrape le temps perdu... »

Déclaration qui rejoint, on l'aura compris, le message d'ouverture du juge au sujet de l'espoir d'une paix sociale retrouvée grâce au travail de la mémoire.

31 La menace d'un possible retour au passé, que jusqu'en mars 1998 l'on pouvait qualifier de réelle, dans la mesure où Pinochet restait au commandement de l'armée chilienne, est par ailleurs un des discours qui va véhiculer l'option du "consensus » dont on parlait dans la première partie de cet article. Avec l'arrestation de Pinochet et malgré la déception pour le résultat final, ce consensus de la peur fera crise comme l'exprime dans $E l$ caso Pinochet Carmen Hertz (avocate des victimes), permettant une évolution historique de la démocratie au Chili :

Pareciera que a espaldas de todos nosotros, a espaldas de la sociedad chilena, hubo una especie de acuerdo de otorgarle garantías de impunidad al genocida que es Augusto Pinochet. La posición que asumió el gobierno de defensa de principios y no de personas, es decir, enarbolando el tema de la soberanía como un argumento contra la acción de la justicia internacional, es una barbaridad. Ahora, creo que la detención de Pinochet provocó un cambio en la sociedad chilena y todas las encuestas de opinión que se hicieron señalaban que 
el 70\% de la población decía que Pinochet tenía que ser juzgado. Hay una inconsecuencia que sólo puede ser entendida en el marco de cómo se ha desarrollado la transición a la democracia en Chile que ya lleva largos diez años, en un proceso que cuesta mucho entender, en relación sobre todo con el tema de nuestra memoria histórica que significó en definitiva mantener toda la institucionalidad de la dictadura, con reformas que no afectaron jamás la esencia de esa institucionalidad, eso es lo tremendo y eso es lo que hizo crisis, hace crisis ahora, a fines de los noventa y es lo que la detención de Pinochet, de alguna manera, dejó al desnudo ( 1 h 31 min $02 \mathrm{~s}-1$ h 32 min $46 \mathrm{~s})^{18}$.

Dans ce sens-là on peut parler, en effet, d'une transformation des pratiques sociales qui vont quitter la sphère marginale pour s'exprimer dans la rue, devenue alors scène publique d'une exigence de justice et de vérité. Scène de dénonciation, également, d'un manque de cohérence - voir de complicité - des gouvernements et de la classe politique face aux crimes de la dictature.

En tant qu'espace ouvert à la parole et à la dénonciation, le documentaire devient le miroir où cette transformation (et la nécessité de son total accomplissement) se reflète et peut être regardée (interrogée) car, au-delà de la démonstration des faits, comme l'on expliquait plus haut, le documentaire offre un discours d'ouverture à la réflexion et d'exigence de changement qui dépasse la pure création du film en tant que récit narratif d'un événement.

Dans l'épilogue de son documentaire, la caméra se pose sur le visage de deux victimes de la torture (Nelly et Gabriela) qui, se réjouissant de leur revanche (être vivantes, pouvoir parler...), apportent la conclusion au documentaire :

Yo creo - dit Gabriela - que la fuerza de la memoria es algo que nos permitirá sanar... por eso es que es tan importante establecer la memoria colectiva, para poder vivir ahora y poder construir el futuro (1 h $46 \mathrm{~min} 07 \mathrm{~s})^{19}$.

La nécessité de la récupération de la mémoire, en tant que condition sine qua non pour construire un avenir où les démons du passé seraient en fin guéris, représente donc la conclusion du documentaire de Guzmán qui, rangé aux côtés des victimes, contribue à briser à son tour le discours consensuel (et la logique de l'action) officiel auquel il s'oppose.

La lecture que le documentaire de P. Guzmán fait de l'arrestation de Pinochet à Londres semble aller donc dans le sens d'un progrès décisif dans la transformation de la société chilienne. Elle représenterait l'ouverture d'une nouvelle voie vers la consolidation d'une démocratie plus juste et authentique. El caso Pinochet rappelle cependant que seul un véritable travail de la mémoire pourra rendre effective la continuation de cette dynamique initiée à Londres.

Marcela Said, pour sa part, offre dans son documentaire I love Pinochet un autre aspect de ce que la détention de Pinochet à Londres (et la médiatisation dont elle a fait l'objet) a révélé : la menace que représente, aussi bien pour la consolidation d'une démocratie plus juste que pour une véritable transformation de la société, le soutien des pinochetistas à une dynamique de déni, d'oubli et de mépris vis-à-vis des victimes de la dictature,

Née en 1972, Marcela Said est une jeune réalisatrice qui a vécu le processus dictatorial et le retour à la démocratie depuis « les marges de l'histoire ", pourrait-on dire, d'abord de par son jeune âge au moment des faits, ensuite pour son installation à Paris en 1995 afin de compléter ses études de cinéma. Elle aurait donc un regard plus distancé, moins personnellement impliqué, même si pas moins engagé : le regard de ces jeunes Chiliens qui, contraints à vivre en dictature, et lassés des promesses jamais accomplies de la jeune démocratie, décident un jour de quitter le pays à la recherche de leur propre voie ${ }^{20}$. 
En 1998 Said est donc à Paris quand elle apprend la nouvelle de l'arrestation de Pinochet, elle décide ensuite de réaliser son film et de l'articuler autour de ce secteur de la population chilienne qui persiste à soutenir le dictateur. Sa démarche est avant tout une démarche compréhensive, elle cherche, en effet, une réponse, qu'elle tente de trouver au fil de son documentaire, à la question: Qui sont les pinochetistas? Ceux qui, encore en connaissance des faits, continuent à soutenir, avec ferveur, le dictateur.

Paradoxalement, ce regard - a priori plus distancé - se traduit dans la création par l'élaboration d'une œuvre qui est foncièrement subjective. Tout d'abord d'un point de vue esthétique, visuel : elle choisit l'emploi d'une caméra subjective qui parcourt les rues du Chili et s'introduit (nous introduit) en témoin dans les maisons, les rencontres, les célébrations des pinochetistes pour mieux les scruter. Le récit est également subjectif dans la mesure où le je de l'auteur s'exhibe à plusieurs reprises dans des interventions qui prennent sens dans ce que la réalisatrice appelle « l'enquête morale » du film.

En effet, au moyen d'une structure dialectique, le portrait des pinochetistes, base narrative du film, s'articule autour d'une série de questions et de conclusions de la réalisatrice qui en se posant des questions nous guide vers une réflexion personnelle. Tel que Said l'exprime dans la "note d'intention du film», le portrait que celui-ci réalise des pinochetistes aurait une double implication: d'une part, il cherche à définir la base « idéologique » du soutien à Pinochet, d'autre part, il met en évidence, par la construction de son discours, les conséquences possibles d'une polarisation sociale vue en tant qu'entrave à une construction démocratique véritablement plurielle.

$\mathrm{Au}$ moyen donc de la caméra subjective, la réalisatrice construit le portrait des pinochetistes, nous entraînant au milieu même de cet "univers du partisan pinochetista " (ses motivations, ses raisons, ses valeurs) que le documentaire cherche à cerner. Grâce à cette intrusion dans les espaces de vie des partisans de Pinochet le film montre la base «idéologique » sur laquelle s'établit leur défense. Les différents intervenants (parmi lesquels on retrouve, entre autres, les deux extrêmes de la société chilienne : bourgeoisie et classe populaire, ainsi que quelques intellectuels ${ }^{21}$ ) déploient les arguments qui expliquent leur position: la justification, d'une part, du coup d'État face à la menace communiste et l'éloge, d'autre part, d'une politique économique ultralibérale qui aurait donné naissance à un « nouveau Chili » puissant et moderne.

La caméra recueille les entretiens de Fernando Barros (abogado) et Fernando Moreno Valencia (filósofo), les plans américains en position semi-zénithal les magnifient; debout dans leurs domaines - l'imposante maison familiale pour l'un, le bureau à l'université pour l'autre - ils nous offrent leurs conclusions sur les faits du passé : face à la menace communiste le coup d'État se serait avéré, en effet, inévitable. Ainsi le coup d'État représente une sorte de geste libérateur, et devient «horreur fondatrice», comparable d'ailleurs à la Révolution française... (26'09"), incontournable dans la construction d'un nouveau Chili ${ }^{22}$. Une guerre nécessaire de laquelle Pinochet est sorti victorieux «seul et unique vainqueur du communisme dans le monde - dira plus tard l'une des amies de Mónica Salinas - et cela dans une seule journée, tout un exemple de courage et de détermination pour le monde... » (30' 39").

3 Pinochet est ainsi élevé à la catégorie de sauveur selon une conception messianique de la politique que le film ne se prive pas de montrer. Il est désigné, par ailleurs, très affectivement, comme "el tata " (le grand-père) et Jonathan Arcos (un garçon dans l'adolescence) se rappelle les larmes aux yeux, devant le plan serré de la caméra, de la 
première fois où il a vu "son général » et explique son émotion: Pinochet est "presque comme mon papa, je crois qu'il m'a presque donné la vie... (soupir) » (33' 20").

Un autre moment de ce type de paroxysme a lieu quand la caméra parcourt les salles de la fondation «Président Augusto Pinochet Ugarte » (destinée à la transmission et la défense de ses valeurs et de son œuvre : 11' 14"). Arrivé face au jardin de style japonais tant aimé par le dictateur, aujourd'hui flétri, le guide commente: "dans la minute où il fut séquestré à Londres, le jardin, aussi... on dirait qu'il est mort » (13' 20")...

Une explication possible de cette déification de la figure de Pinochet nous est offerte par une des amies de Mónica Salinas, dans une des séquences les plus significatives - et ahurissantes - du film (29'02"). Le groupe d'amies prend le thé (la once) dans le salon de Mónica, moment convivial auquel nous sommes conviés : la caméra devient une des convives et participe ainsi de la conversation sans intervenir. L'amie de Salinas, donc, laisse entendre que Pinochet incarne la solution à tous les maux du Chilien : « le Chilien - dit elle - est d'une mentalité médiocre, ce pays est médiocre, donc quand on habite dans des pays médiocres, ils doivent être commandés d'une main ferme, car le Chilien ne fonctionne que sous pression. »

Définition du Chilien dont, bien entendu, les pinochetistes s'excluent, comme l'explique plus tard une autre amie qui, après avoir affirmé « les Chiliens sont une bande d'imbéciles sans aucune reconnaissance...», interpelée par son amie (ils sont...?) déclare avec conviction « ils sont, car je n'appartient pas au lot, je suis "pi-no-che-tis-ta", j'adore mon général Pinochet ", et l'autre amie conclut "ce que les autres ne sont pas des Chiliens, ils n'ont pas de patrie, ils ont un drapeau rouge co-mmu-nis-te... » (30' 13").

Les Chiliens seraient, donc, tous communistes ou pinochetistes, ignorant ainsi les autres positions citoyennes modérées (comme ceux qui, n'étant pas communistes, ou tout simplement de gauche, ne cautionnent pas le coup d'État ni ne considèrent non plus la dictature comme une alternative valable...). Voilà une des bases « idéologiques » qui sert à justifier l'action menée par Pinochet et son armée: le peuple chilien, de par son immaturité ou son ignorance, n'était pas prêt à décider de son destin... Une vision donc paternaliste de la politique qui justifierait l'intervention de l'armée dès que les urnes portent atteinte aux intérêts, notamment économiques, d'une partie des Chiliens.

On a donc un pouvoir qui s'établit par-dessus la légalité démocratique cherchant à imposer par tous les moyens leur idée du Chili, en s'appropriant par ailleurs - comme le montre le documentaire - tous ces symboles (drapeau, hymne, folklore.... ${ }^{23}$, quitte à exclure, dénigrer, et éliminer ses antagonistes et, par la même occasion, la possibilité d'une construction collective.

L'éloge du néolibéralisme ainsi que ce nouveau Chili que Pinochet aurait réussi à construire est le deuxième argument de défense de la figure du dictateur. Grâce à Pinochet, le Chili est devenu une puissance économique de l'Amérique latine et un exemple pour le continent, y compris pour les États-Unis, explique Enrique Jara (candidat de l'UDI) (39').

L'étendue, encore aujourd'hui, de ce discours fallacieux, selon lequel la dictature aurait permis au Chili de devenir un pays riche (la pauvreté au Chili atteignait un taux de 38,6\% en 1990, réduit en 2006 à 13,7 \%, l'indigence ayant également baissé de $13 \%$ à 3,2 \% ${ }^{24}$ ), est d'ailleurs une de raisons qui pourraient expliquer le soutien des classes populaires au régime militaire. 
51 Ce soutien, obtenu au moyen d'un discours qui jongle entre néolibéralisme et populisme, est fondamentalement basé sur l'idée que le pays a réussi sa course vers la " modernisation ", menée à bien par le régime militaire. Cette réussite ferait du Chilien ce que Tomás Moulian (1997, $99 \mathrm{~s}$ ) appelle un citoyen «credit-card» et de la « consommation de masse » sa doctrine d'intégration.

Marcela Said elle-même prend la parole pour expliquer l'importance que la propagande de Pinochet a eue dans la construction d'une image de la dictature en tant que garantie de la réussite économique. Réussite qui se veut, par ailleurs, valeur sûre du nouveau Chili que le régime dictatorial avait cherché à construire.

Dans la séquence suivante ( $\left.36^{\prime} 13^{\prime \prime}-37^{\prime} 04^{\prime \prime}\right)$ les images d'archives de la bande publicitaire de la campagne du « oui », pendant le plébiscite de 1989, s'introduisent d'abord dans le récit; on continue ensuite à entendre la mélodie de la bande publicitaire tandis que les images d'archives laissent leur place à celles de la voiture parcourant les rues de grands centres commerciaux de Las Condes (symbole du pouvoir économique ultralibéral dans un des beaux quartiers de Santiago). À ce moment-là, Marcela Said s'exprime :

Durante diecisiete años la imagen de Pinochet inaugurando casas para los pobres, fue tal vez la imagen más repetida de la televisión chilena. La dictadura no estuvo privada de propaganda, que día a día nos trataba de convencer de lo bueno y fructífero que era el gobierno militar ${ }^{25}$.

$\mathrm{Si}$, avec la dictature, les libertés publiques se réduisent, si la capacité du peuple à participer à la vie publique et à décider de son destin en tant que nation disparaît, cela procure en compensation l'accès à la consommation de biens des pays « développés » et la possibilité pour le Chili de devenir un pays «moderne». L'intervention de Patricia Maldonado dans une réunion des partisans de Pinochet illustre nos propos: elle interpelle son public et demande (34' 49") «Que se serait-il passé si dans ce pays il y avait eu un régime communiste? Serait-on réunis aujourd'hui ici? Pardon, aurais-tu ta voiture ? Aurais-tu ta télé en couleur ?...»

Suivi par la caméra dans sa déambulation au long des trottoirs étroits et démunis de son quartier populaire, la voix d'Israel Arcos conclut: «La grande erreur du général fut d'abandonner le pouvoir... s'il n'avait pas quitté le pouvoir, on serait tous riches aujourd'hui » (47'30")...

Le documentaire met ainsi en scène, d'une part, le portrait d'une partie de la société chilienne qui, faisant fi des pertes humaines, exhibe tout un éventail de justifications et d'arguments pseudo-idéologiques pour montrer l'évidence même de leurs convictions. Une partie de la société chilienne qui, après avoir accumulé les richesses du pays et contrôlé toutes les sphères du pouvoir pendant des années, rencontra, en même temps, un ennemi à combattre, des alliés dans la classe populaire ou moyenne (qui aspirent à devenir un jour aussi riches qu'eux), et un héros pour les sauver, ils seront désormais pinochetistes et combattront le communisme...

D'autre part, l'enquête morale continue et à la première question (Comment, en connaissance des faits, peut-on continuer à justifier la violence d'État exercée par les militaires?) s'ajoute une deuxième non moins fondamentale: Comment la société chilienne peut-elle rester impassible face à une telle situation? Car la justification et la passivité constituent le discours et le mode d'(in)action, et c'est cela le plus troublant comme semble alerter le documentaire, non pas d'un groupuscule extrémiste en manque total de lucidité mais d'une partie trop importante de la population chilienne qui, soit par 
ignorance, soit par désinformation, par intérêt, mépris ou indifférence, cautionne cette posture. C'est donc la réalisatrice qui s'empare à nouveau du récit et s'interroge :

En 1988 Pinochet llama a plebiscito, después de dieciséis años de régimen militar un 44\% de chilenos se déclarèrent abiertamente pro-Pinochet; en 1999 el candidato de coalición de derecha Joaquín Lavín, quien en 1988 llama a los chilenos a votar por Augusto Pinochet obtiene un $47 \%$ de los votos. Resulta preocupante constatar que un heredero directo de la dictadura se anuncie como el futuro presidente de Chile y más inquietante lo es el hecho de que todos los sectores de la sociedad, sin distinción sean permeables a esas opiniones $\left(15^{\prime}-15^{\prime} 58^{\prime \prime}\right)^{26}$.

Cette articulation dialectique entre le portrait présenté et la réflexion de la réalisatrice semble nous conduire à la nécessité de nous interroger à notre tour sur les implications de ce soutien à un régime qui laissa derrière lui, non seulement des milliers de morts, de disparus, de torturés, de familles brisées... mais aussi une société divisée et incapable de se rassembler encore aujourd'hui dans la construction collective (et non pas partisane, sectaire) d'un avenir démocratique pour tous ${ }^{27}$.

Marcela Said met finalement en cause dans son documentaire une démocratie qui accepte les règles du jeu des militaires et exclut une bonne partie des Chiliens; une démocratie illusoire dont la transition s'avère un processus infini. Dans l'épilogue de son film la voix off de la réalisatrice pointe le maintien de la Constitution de 1980 comme la véritable victoire de Pinochet, « une constitution - affirme-t-elle - pratiquement inamovible qui réussit à s'imposer comme le résultat d'un pacte pour rentrer dans l'infini processus de la transition. La démocratie se transforme, ainsi, en une illusion pour ceux qui, comme moi, avons cru en elle».

Bien que, comme on l'a vu dans la première partie de l'article, la persistance des institutions de la dictature, vingt ans après sa fin, puisse mettre en cause la consistance des fondements de la démocratie chilienne, les historiens, analystes, sociologues... semblent s'accorder sur le caractère théoriquement abouti de la transition ${ }^{28}$. Or cette période transitionnelle donne lieu non pas à une démocratie solide et sans ambiguïté mais à une nouvelle étape (transition de la transition?) de construction démocratique où l'objectif serait d'arriver à ce que, au-delà des idéologies, les Chiliens puissent tous se rassembler dans un projet commun, collectif, de défense des principes démocratiques, ce qui constitue la base, le pilier de la durabilité et de la solidité de toute démocratie.

C'est cette idée que semble vouloir transmettre, en définitive, Marcela Said dans son documentaire. La persistance d'une logique belliciste, héritière de la guerre froide (et d'une simplification effrayante), le maintien, peut-on dire, anachronique de la polarisation idéologique, et en général la répétition sans aucune distance critique des discours implantés pendant la dictature, rendent compte d'un trop grand attachement au passé qui met en doute leur foi même dans la démocratie d'un secteur important de la population chilienne. Voilà ce qui fait défaut à la démocratie chilienne et qui représente, peut-être, un de ses plus grands défis pour l'avenir : le changement de mentalités.

En définitive, ce que nous nous efforçons de souligner ici est qu'au-delà de - ou peut-être aussi, en raison de - la qualité artistique de deux films, aussi bien El caso Pinochet que I love Pinochet présentent un intérêt historique particulièrement remarquable. Et cela en deux sens complémentaires. Ils s'établissent, tout d'abord, en tant que trace documentaire; ils cherchent ensuite à analyser, expliquer, contester, dénoncer, intervenir dans les faits racontés afin de provoquer le débat, incitant à une réflexion. 
caso Pinochet, qui, comme on l'a vu, alterne le récit des faits et celui des victimes, se veut espace d'expression d'une mémoire blessée et devient trace documentaire des témoignages: espace d'une mémoire déclarée. I love Pinochet pour sa part réalise un portrait des partisans de Pinochet qui deviendra trace documentaire de leur pensée.

63 jouent également un rôle de médiateur entre mémoire et Histoire (Ricœur, 2000, p. 215), dans la mesure où les témoignages, une fois enregistrés, quittent « la sphère orale », donc privée, et deviennent (potentiellement) publics, ou, ce qui revient au même, documents à valeur historique pouvant être interrogés par un historien « dans la pensée d'y trouver une information sur le passé » (Ricœur, 2000, p. 226).

Mais, au-delà de cette fonction médiatrice, les documentaires de Marcela Said ou Patricio Guzmán ont une tout autre valeur, donnée par l'articulation de leur double dimension esthétique et éthique. Ce qui leur confère des possibilités de réception multiples.

En effet, le traitement de l'image, la recherche esthétique, qui différencient ces documentaires $\mathrm{du}$ documentaire purement «journalistique», sont foncièrement solidaires d'une dimension éthique exprimée par la mise en scène d'un je, le je de l'auteur, qui s'aventure dans la recherche et la construction d'un nous. Un nous auquel ce je appartient, avec qui il s'identifie ou bien qu'il questionne. C'est dans ce sens que le documentaire doit être vu et estimé en tant que document pour l'Histoire.

$\mathrm{Si}$, en tant que document sur l'Histoire, les documentaires proposent, en "montrant " l'Histoire, en «parlant» d'elle, une analyse des réalités sociales, ils participent, également, d'une dynamique de questionnement sur les faits historiques. C'est cela qui les transforme en documents pour l'Histoire, car ils peuvent alimenter (et intervenir dans) la lecture de l'Histoire.

Comme on l'a dit, par le recueil de témoignages, ils deviennent traces documentaires. Par leur obstination, ils encouragent leur public à l'insoumission contre le silence imposé et deviennent ainsi acteurs de la résistance. Ils seront, finalement, amenés à avoir un rôle primordial pour les nouvelles générations, tel que l'exprime Patricio Guzman lui-même dans une des réponses de l'entretien qui suit mon article et qui nous servira de conclusion :

Yo creo que en general las cosas van progresando lentamente, porque grandes sectores de la sociedad trabajan los temas de la memoria y el propósito de ocultar completamente todo lo que pasó durante la dictadura no ha conseguido concretarse (a pesar de la derecha y la concertación) y que, con el tiempo, los jóvenes seguirán buscando esas informaciones que nunca tuvieron y en ese momento los documentales seran una « memoria viviente ${ }^{29}$. 


\section{BIBLIOGRAPHIE}

BAROZET Emmanuelle, « Les élections de 2005-2006 au Chili : Après la transition, quelle démocratie ? », dans Georges Couffignal (dir.), Amérique latine, les surprises de la démocratie, Paris, La Documentation française, 2007, p. 21-33.

D’HENNEZEL Claire (Observatoire des Amériques), « Le Chili de Michel Bachelet : la première année de pouvoir » [en ligne], La chronique des Amériques, $\mathrm{n}^{\circ}$ 9, mars 2007, disponible sur <http:// www.ieim.uqam.ca/IMG/pdf/chro_d_Hennezel_07-09.pdf> [consulté le 9 juillet 2010].

KRITZ Neil J. (sous la dir. de), Transitional justice. How Emerging Democraties Reckon With Former Regimes, 3 vol., Washington DC, United States Institute of Peace Press, 1995.

LEFRANC Sandrine, Politiques du pardon, Paris, PUF, 2002.

-, « Commissions de vérité : un “injuste” modèle de justice? », dans L'État du Monde 2009, Paris, La Découverte, 2008.

Lово Olga, « La mémoire obstinée de Patricio Guzmán. Le passé comme (re)construction du futur », Tigre, $n^{\circ} 14$, « La Trace 2 : Trace et Histoire », George Tyras (éd.), CERHIUS, ILCEA, université Stendhal-Grenoble 3, 2006, p. 193-210.

Moulian Tomás, Chile Actual, Anatomía de un mito, Santiago du Chili, Lom éd., 1997.

RICHARD Nelly, « La transition politique au Chili : formalisme démocratique et déchirure de la mémoire », dans Alain Bossat et Jean-Louis Déotte, L'époque de la disparition. Politique et esthétique, L'Harmattan, 2000, p. 175-183.

RICCEUR Paul, La mémoire, l'Histoire, l'oubli, Paris, Seuil, 2000.

\section{ANNEXES}

\section{Entretien a Patricio Guzman}

1. ¿Qué le empujó a hacer un filme sobre el proceso de detención y enjuiciamiento frustrado de Pinochet?

El sólo hecho de leer en el periódico que Pinochet podría ser sometido a juicio me impulsó inmediatamente a hacer el film. Aunque después supe que sería el primer dictador juzgado en la historia después de Nuremberg, en el primer momento no me planteé ninguna reflexión sino sencillamente seguir el impulso de hacer la película. À mi modo de ver no fue un proceso frustrado pues fue un ensayo general para lo que hoy es el Tribunal Penal Internacional. Por otra parte, gracias al encierro de Pinochet en Londres, por primera vez, la gente perdió el miedo en Chile.

2. Una vez (en una conferencia en Lyon) le oí decir que la democracia había empezado verdaderamente en Chile con la detención de Pinochet, ¿podría desarrollar esta idea?

Aunque yo lo haya mencionad alguna vez, esto no es en realidad estrictamente cierto, pues la transición comenzó cuando Pinochet perdió el plebiscito en Chile y se consolidó con la llegada al poder de Aylwin. Pero fue el proceso de Londres lo que influyó para 
expulsar a Pinochet del senado, para quitarle su inmunidad parlamentaria, su arresto domiciliario, etc. Esto fue lo que derrumbó al tirano, demostró que no era invulnerable y aceleró la transición.

3. À diferencia de otros documentales suyos (como Salvador Allende) usted tiende a «borrarse» en El caso Pinochet, ¿es una opción de búsqueda de objetividad... casi como la «puesta en escena» de un «reportaje»?

Los largos planos a las víctimas que yo interrogo, aunque en realidad son planos clásicos (generalmente de la cintura para arriba), ninguna televisión del mundo se atrevería a filmar o producir, porque las televisiones tienen horror al silencio, a las vacilaciones del entrevistado, al tiempo sostenido. Estas entrevistas atraviesan la frontera que hay entre el periodismo y el documental, y se convierten en un espacio cinematográfico, en secuencias cinematográficas.

4. Su obra tiene una importancia indiscutible en el marco internacional y un papel históricotestimonial importante, ¿qué pasa con Chile?: El caso Pinochet iha sido distribuido en Chile? ¿Se han difundido alguno de sus documentales en la televisión, en centros educativos? si no, ¿qué quiere decir esta «negativa»?

Ninguna película mía ha sido comprada por ninguna televisión abierta de Chile. Ellos dicen que yo les cobro muy caro, porque me niego a aceptar sus precios escandalosos (ellos suelen pagar más o menos 2000 dólares por cada documental).

El único filme mío distribuido profesionalmente en salas de cine ha sido «Salvador Allende», que hizo 65000 espectadores (la cifra, aunque pequeña, es la más alta hecha por un documental en Chile; en Francia sobrepasó los 125000 espectadores).

«El caso Pinochet» hizo 80000 espectadores en México, pero en Santiago solamente permaneció 2 semanas en una sala pequeña y fue sacada de la cartelera ante la llegada de Harry Potter.

Los distribuidores de cine en Chile son muy pocos (son cinco o seis personas), todos muy conservadores, que jamás han sentido simpatía hacia los documentales y menos hacia la memoria histórica. La única excepción han sido los cines Hoyts, durante los primeros tiempos de esa compañía. Los jóvenes distribuidores de «Allende» fueron otra excepción y esa pequeña empresa ya no existe.

Durante un tiempo existió un paquete con 4 VHS (las tres «Batallas» y «La memoria obstinada»). Pero consumido el stock no se hizo una reedición. En este momento hay el proyecto en el futuro de editar un DVD con todas mis obras. Pero yo no me lo creo hasta tenerlo en mis manos.

5. A lo largo de los últimos años el cine documental vive un auge relativo del que usted es en gran parte responsable. Recientemente asociaba usted salud democrática y salud del documental ¿En qué sentido, según usted, y qué papel ha jugado-juega el cine documental (en Chile concretamente) en el desarrollo de un cambio social hacia la democracia ?

Es muy saludable que haya 15 o 20 buenos documentalistas y que muchos planteen los temas de la memoria histórica o que analicen la situación actual. Fondart proporciona el dinero. Pero ni las televisiones ni las salas les acogen bien. Es decir, es un movimiento documental que no tiene una distribución apoyada por el estado.

6. Actualmente finaliza su último trabajo, en un contexto político-social completamente distinto al de El Caso Pinochet. El gobierno de Bachelet ha significado la esperanza para muchos chilenos y ha hecho avanzar las cosas (en materia de derechos humanos también). Muchos (entre otros el cine documental) siguen en cambio su lucha contra la 
impunidad: En vista de los cambios de los últimos años ise puede hablar de una democracia plenamente consolidada?

Siento una gran decepción con el gobierno Bachelet. Durante su mandato una documentalista (Helena Varela) ha sido encarcelada y acusada de terrorista por estar filmando una película sobre los Mapuches. Durante su gobierno otra cineasta documentalista chilena (Valentina Palma) fue apaleada en México, vejada sexualmente y expulsada hacia Chile por estar filmando una manifestación contra la policía mexicana en un pueblo cerca de la capital. Aunque yo le entregué un dossier a la ministra de la cultura sobre este caso, el gobierno no hizo mucho por ayudar a esta joven. Dentro de poco la Concertación quiere hacer aprobar un texto sobre los «derechos de autor» que autoriza a las televisiones a utilizar planos que aparecen en nuestras películas sin pagar los derechos. Es un verdadero robo legal, aunque se espera que el texto original sea corregido.

Saliendo del cine documental (que es un espacio muy pequeño) yo noto en general un enorme vacío, una enorme ausencia de proyecto en la Concertación, un agotamiento y a la vez síntomas de corrupción.

7. En un supuesto contexto favorable al cambio, ¿el compromiso del cine documental y del arte en general con dicha lucha, sigue vigente?

Yo creo que en general las cosas van progresando lentamente, porque grandes sectores de la sociedad trabajan los temas de la memoria y el propósito de ocultar completamente todo lo que pasó durante la dictadura, no ha conseguido concretarse (a pesar de la Derecha y la Concertación) y que, con el tiempo, los jóvenes seguirán buscando esas informaciones que nunca tuvieron y en ese momento los documentales serán una «memoria viviente».

\section{I love Pinochet : note d'intention de Marcela Said}

Il y a quatre ans, j'ai quitté mon pays d'origine, le Chili, parce qu'il m'était devenu simplement insoutenable d'y vivre. Comment expliquer ces grandes petites choses, comment parler de la fragmentation d'un pays, de la perte de ses valeurs essentielles, du fascisme ordinaire, de ce malaise qui me poursuit jusqu'ici ? C'est un ensemble d'impressions où se mêlent peur, haine, tristesse et impuissance.

Je m'appelle Marcela, j'ai 28 ans et j'ai grandi avec la dictature de Pinochet. Cette dictature qui a réussi à transformer une société démocratique, civilisée et républicaine, en un pays scindé en deux : un Chili qui rêve de justice sociale et d'une vraie démocratie, l'autre, le Chili de Pinochet, qui se vante sans cesse d'un progrès économique géant et essaie de justifier par tous les moyens les méthodes employées par Pinochet, dont la torture.

La fragmentation est partout, dans les plus infimes détails, dans les quartiers, les lieux de rencontre, les écoles, à l'université... Tout est séparé en deux parties, en deux pensées. Jusqu'à quand nous sera-t-il possible de coexister ainsi ?

Au Chili, le concept de souveraineté populaire a un caractère tout relatif, car la majorité est à la disposition d'une minorité aisée qui détient le pouvoir économique, et de facto politique dans un Chili entièrement privatisé, où une toute-puissante Église catholique a épousé la « théologie ultra-libérale ». Un pays qui a perdu sa laïcité n'est plus une république. Le Chili est l'un des rares pays du monde où il n'y ait pas une loi de divorce, et où l'avortement même thérapeutique est passible de prison. 
Mais qui sont donc ces gens-là qui protègent et vénèrent Pinochet avec tant d'acharnement? Qui sont les pinochetistes ? Une bande de néo-libéraux fascisants qui portent une croix autour du cou et influencent encore notre gouvernement ? Ou les victimes de l'opérette Pinochet, de la manipulation médiatique? Ce qu'on ne peut pas négliger de considérer, c'est que beaucoup des hommes qui ont travaillé aux côtés de Pinochet étaient parmi les plus brillants cerveaux que comptait le Chili. Étrange, non?

\section{Naissance d'un projet}

Quand on a arrêté Pinochet à Londres, en octobre 1998, j'ai cru voir apparaître une lueur d'espoir. Finalement les choses allaient changer pour toujours. La situation à Londres était singulièrement proche de la réalité chilienne, avec d'un côté les victimes, et de l'autre, les pinochetistes qui avaient entrepris un long voyage pour venir soutenir leur cher général. Combien de mes amies étaient là, du côté de Pinochet ? Comment, sachant toutes les horreurs commises, pouvaient-elles lui donner leur soutien inconditionnel ? C'est à ce moment-là qu'a germé en moi l'idée d'entreprendre un documentaire qui tenterait de donner une idée de l'ampleur et de la complexité du phénomène pinochetiste au Chili. Mais la tâche serait ardue, et peut-être pénible. J'avais devant moi une longue période de réflexion.

Après avoir vaincu la peur de possibles représailles et l'idée de me voir confrontée à mes amies et ma propre famille, car mon père persiste à défendre Pinochet, je me suis décidée à me rendre chez les pinochetistes. Il me fallait m'infiltrer à la fondation Pinochet, le lieu privilégié de leurs réunions. Au départ, j'avais le profil qu'il faut pour les approcher... Issue d'une bonne famille, je possède d'emblée l'image susceptible de vaincre la méfiance, à leurs yeux, je pourrais faire partie du groupe.

En décembre 1999, je suis partie pour deux mois, avec une petite caméra DV, pour réaliser mes premiers contacts. Non sans difficulté, j'ai réussi à approcher mon premier personnage, Monica Salinas, arguant de l'importance de montrer dans le monde sa vision pinochetiste des choses. Je voulais vraiment comprendre leur pensée et les raisons qui sont les leurs pour soutenir Pinochet...

Au mois de mars, je suis retournée au Chili, pour continuer mon travail de repérage et commencer à tourner les premières images de mon film...

\section{Introduction historique du film}

Le 11 septembre 1973 fut une date noire dans l'histoire du Chili moderne. La junte militaire a pris le pouvoir dans la barbarie, pour le conserver par la terreur. Ce jour-là, Allende s'est donné la mort, la Moneda a brûlé et avec elle tous les rêves de la révolution démocratique chilienne se sont évanouis. Le bilan d'une commission paritaire de 1991 fait état de 3198 morts sans compter les milliers de victimes de tortures. Comment expliquer, après les erreurs et les horreurs commises, la permanence du phénomène pinochetiste, et quelle est l'importance qu'on doit lui attribuer? Il y a peu, l'élection de Jorg Haider a frappé l'opinion européenne et mondiale. L'Autriche, au mépris des leçons du passé, a porté au pouvoir le parti d'un héritier du fascisme. Le cas Haider invite à poser un regard critique sur ces peuples qui semblent oublier leur propre histoire. En décembre 1999, des élections ont eu lieu au Chili. Le populiste d'extrême droite Joaquin Lavin, ancien collaborateur de Pinochet, y obtint $47 \%$ des suffrages, avant de donner une accolade empoisonnée à son adversaire victorieux. Dans les dix années à venir, le Chili pourrait être un parfait candidat pour un gouvernement d'extrême droite. Mais qu'est-il arrivé, 
qu'arrive-t-il encore au peuple chilien ? Est-ce le même peuple qui, en 1970, a séduit les démocrates du monde entier, qui ont vu dans l'expérience réformatrice issue des urnes un espoir pour la social-démocratie en Amérique du Sud ? La société chilienne a connu de grands changements depuis 1973. Devenu ultra-libéral et globalement plus prospère, mais encore plus inégalitaire qu'auparavant, le Chili a préservé ses traits conservateurs, par le biais notable d'une puissante Église catholique (60 \% de l'éducation est prise en main par l'Église). L'éducation supérieure est réservée aux nantis, tout comme la santé publique, la précarité est partout... Dans ce contexte, le Chili me semble parfois présenter une vacuité intellectuelle inquiétante. Ce qui paraît le plus étonnant, en effet, c'est l'indifférence des Chiliens face à cette situation. Le Chili est un pays malade et amnésique. Refusant les complexes d'un examen de conscience, une partie du pays enfouit son passé en élevant à Pinochet une statue de sauveur de la nation. Si les victimes et leurs familles cherchent à la mettre à bas, la majorité des Chiliens détourne son regard. L'activisme des partisans de Pinochet est le symptôme le plus visible de la maladie qui affecte aujourd'hui mon pays : l'inconscience sociale et politique.

\section{Le film}

C'est un film sur le Chili actuel. Un regard porté sur le système social et politique légué par Pinochet, dévoilé à travers les portraits de pinochetistes, ces gens qui par milliers et toutes classes confondues croient en la pérennité d'un système autoritaire et ultralibéral, teinté de féodalité. Leurs discours ont en commun de justifier la répression brutale du passé par la nécessité impérieuse d'alors de rétablir l'ordre, de sauver le pays de la « menace socialiste ». On découvrira à quel point ce système de pensée est ancré dans la société chilienne actuelle et risque encore de perdurer malgré les avancées récentes de la justice. Le film sera aussi une enquête morale dont la question de départ sera de savoir comment, en connaissance des horreurs commises, ces gens peuvent s'affirmer encore aujourd'hui « pinochetiste ». C'est par une démarche compréhensive, bien qu'en aucun cas relativiste, qu'on entend tenter d'éclairer le problème. En somme, c'est un film qui dénonce ce qui dans la machine pinochetiste n'a pas cessé de fonctionner avec le retour de la démocratie, jusqu'à franchir l'écart des générations.

\section{NOTES}

1. En 2005 le président Ricardo Lagos apporta une première modification à la Constitution de 1980. Par la loi 20.050 il élimine 58 articles de la Constitution et remplace la signature de Pinochet par la sienne. Cette première modification de la Constitution de 1980 met fin, entre autres, à l'imposition de sénateurs "désignés » ainsi qu'à toute tutelle des forces armées sur la politique et marque le signe d'un début de rupture avec le passé institutionnel imposé par les militaires.

2. On pourrait mentionner également la loi d'amnistie ou la loi antiterrorisme promulguées par la junte militaire en 1978 (loi d'amnistie, décret-loi 2191) et 1979 (loi antiterrorisme, décret-loi 2621). La loi d'amnistie suppose un empêchement au processus de justice ouvert contre la violation des droits de l'homme pendant la dictature. La loi antiterrorisme a été appliquée dernièrement à l'encontre des Indiens qui cherchent à protéger leurs terres, ou même, comme l'explique Patricio Guzman dans l'entretien qui suit mon travail, contre la documentaliste Helena Varela qui a été arrêtée et accusée de terroriste pendant le tournage d'un documentaire sur les Mapuches. Lors de l'Examen périodique universel, effectué le mois de mai dernier par le Conseil des droits de l'homme, l'organisme des Nations unies recommande, entre autres, l'annulation de 
ces lois comme condition sine qua non de la réussite définitive de la transition démocratique au Chili. (Tous les documents peuvent être consultés sur <http://www.ohchr.org/EN/HRBodies/ UPR/PAGES/CLSessions.aspx> ; pour les conclusions de la commission voir aussi : <http:// www.scribd.com/>).

3. En ce qui concerne la loi électorale, la présidente Michèle Bachelet a présenté un projet de reforme qui donnerait dans l'avenir l'opportunité de voter à quelque 4 millions de Chiliens dans le pays ainsi qu'aux Chiliens résidents à l'étranger. Elle cherche, par ailleurs, à supprimer le système binominal et à redéfinir les circonscriptions et les districts en vue d'une plus juste proportion entre le nombre des députés et des sénateurs et les populations qu'ils représentent. Pour mener à bien cette réforme, Bachelet a mandaté en 2006 une commission (connue comme Commission Boeringer) pour la modernisation de l'État. La commission a rendu son rapport le 7 juin 2006. Pour une analyse des premières mesures du gouvernement Bachelet voir: Claire d'Hennezel (Observatoire des Amériques), «Le Chili de Michel Bachelet: la première année de pouvoir», La chronique des Amériques, $\mathrm{n}^{\circ}$ 9, mars 2007, consultable sur <http:// www.ieim.uqam.ca/IMG/pdf/chro_d_Hennezel_07-09.pdf>. Pour une explication du système binominal claire et précise, voir Barozet, 2007, p. 28.

4. En matière de protection des droits fondamentaux le Conseil dénonce, dans le rapport cité, l'absence d'une figure ou entité autonome pour la défense des citoyens ainsi que l'absence d'une institution des droits de l'homme au Chili. En 2007, grâce à la « commission Valech ", un accord a été signé avec les députés de l'opposition (Chadwick/Espinosa) pour permettre l'approbation d'un Institut des droits de l'homme qui n'aura, cependant, de compétence pour exercer des actions judiciaires. Cet accord peut être considéré comme un premier pas pour que le respect des droits de l'homme devienne une « politique » d'État, tel que l'anticipait le discours du président Lagos «No hay mañana sin ayer» en 2003 (voir: <http://www.lanacion.cl/p4_lanacion/ antialone.html>). Le Conseil relève également l'absence d'une législation interne adaptée au standard établi par la communauté internationale.

5. Hugo Gutierrez, avocat des droits de l'homme, déclarait le 25 avril dernier à "Andalucia Solidaria » : «La circunstancia de que hoy siga vigente la constitución política es un hecho demostrativo de la legitimidad que la transición en Chile le ha dado a todo lo creado por Pinochet [...] también existe una continuidad en materia cultural y una continuidad en la impunidad a los violadores de los Derechos Humanos que campaban en Chile hasta la detención de Pinochet en octubre de 1998, cuando el estado chileno se ve obligado por la comunidad internacional a juzgar a Pinochet en Chile.» (<http:// www.andaluciasolidaria.org>.)

6. En matière de droit international, on parlera désormais d'une "justice transnationale », sous le signe des droits de l'homme et de " globalisation » de la loi criminelle. Jack Straw dira dans un communiqué : "Los que comenten abusos contra los derechos humanos en un país no pueden dar por hecho que estarán a salvo en otro. » À ce sujet, voir, entre autres: N. Roth-Arriaza, "The Pinochet effet: Transnational justice in the Age of Human Rights", Philadelphie, University of Pennsylvania Press, 2005.

7. <www.memoriaviva.com>.

8. $<$ http://www.vicariadelasolidaridad.cl/>.

9. $<$ http://www.codepu.cl/>.

10. <www.fasic.org>.

11. En 1990 le gouvernement de Patricio Aylwin met en place la «Commission de vérité et de réconciliation " (Commission Retting) pour établir la «vérité ». Elle a été suivie en 1992 par la "Corporation de réparation et de réconciliation». En 1993 un «Mémorial des victimes » est également inauguré au cimetière de Santiago. Ce n'est cependant qu'après la détention de Pinochet à Londres que le dialogue avec les forces armées entre en jeu: Pérez Yoma, ministre d'Eduardo Frei, promulgue en 1999 une série de «tables rondes» (mesas de diálogo) avec représentation des forces de l'ordre afin d'établir, à nouveau, la "vérité », bien que sans suite 
judiciaire (puisque un secret de 50 ans concernant les noms des responsables est exigé) ; à ces commissions s'ajoute la même année, la « Commission Valech » qui cherche à établir la vérité en matière de Prison politique et torture. En 2004 le gouvernement de la concertation reconnaît la torture comme " politique d'État » pendant la dictature et le droit des victimes à la réparation et reconnaissance morale. Le général J. Emilio Cheyre (chef de l'armée) reconnaît aussi la responsabilité institutionnelle de l'armée dans la violation des droits de l'homme. Plus récemment, le gouvernement de Bachelet déclare le 30 août «journée nationale du détenu disparu ", et en 2007 le Patio 29 du cimetière de Santiago devient monument national. La même année Bachelet annonce la création d'un musée de la mémoire pour fin 2009. Par ailleurs, en mars 2009 le Sénat a refusé un projet de loi contre le génocide, les crimes de guerre et contre l'humanité. Il existe également un projet de loi qui permettrait de réinterpréter l'article $93 \mathrm{du}$ code pénal pour exclure la responsabilité pénale de la loi d'amnistie.

12. Patricio Guzman explique dans l'entretien que je reproduis en annexe de mon article le refus des télévisions chiliennes de diffuser ses documentaires ainsi que le manque de soutien de l'État vis-à-vis de la distribution de documentaires au Chili. L'État, qui débloque des fonds pour aider à la création, ne semble pas pour autant très impliqué dans leur distribution et leur diffusion.

13. Cet essor se traduit de manière concrète non seulement dans la production de films mais aussi dans l'organisation d'événements et la création d'associations qui regroupent les documentalistes et soutiennent leur travail. Ainsi, par exemple, en 1997 Patricio Guzmán crée à Santiago le «Festival international de documentaires »; en 2000 un groupe de documentalistes crée l'association ADOC pour impulser la création de documentaires au niveau national. En Europe et ailleurs les noms de Patricio Guzmán, Marcela Said, Carmen Luz Parot et Carmen Castillo attirent le public et se font entendre dans des festivals internationaux...

14. Parmi les derniers documentaires sur l'Histoire récente du Chili on peut souligner (mis à part les documentaires de Patricio Guzmán) : La alegría de los otros de Ingrid Ormeño, Kika Vadés et Carolina Espinoza (2008) sur le plébiscite de 1988, Actores secundarios de Pachi Bustos et Jorge Leiva (2004) sur le mouvement des lycéens des années 1980, Calle Santa Fe de Carmen Castillo (2008) sur Miguel Henríquez, fondateur du MIR, Estadio Nacional de Carmen Luz Parot (2002) sur les détenus du stade aujourd'hui appelé Victor Jara, Fernando ha vuelto de Silvio Caiozzi (1998) sur l'histoire d'une famille et sa rencontre avec la dépouille de Fernando, disparu et mort pendant la dictature, Aquí se construye de Ignacio Agüero (2000) sur la destruction d'espaces de vie avec les autres, ou du même auteur El diario de Agustín (2009) sur le rôle du journal El Mercurio pendant la dictature, Reinalda del Carmen, mi mamá y yo de Lorena Giachino (2006), un film sur la mémoire et la récupération $d u$ passé...

15. <http://www.patricioguzman.com/>.

16. La batalla de Chile (1975-1979) et La memoria obstinada (1997) précèdent le film qui nous occupe. Salvador Allende (2004) complétera récemment le travail du réalisateur sur le sujet.

17. Bien qu'elle laisse le plus souvent la place aux témoignages des protagonistes: Carlos Castresana, Joan Garcés, Alberto Garreton interviennent pour raconter et analyser la phase préalable à la détention à Londres. Ernesto Ekaizer, Peter Shaad, Jeremy Corbyn, Norman Lamont et Andy McEntee racontent l'arrestation à Londres. Clue Nicholls, Ronald Bartle, Alun Jones, le processus d'extradition.

18. C'est comme si dans le dos de la société un accord avait été passé pour garantir l'impunité au génocide qu'incarne la figure de Pinochet. La position du gouvernement défendant ses principes, brandissant le thème de la souveraineté comme un argument contre l'action de la justice internationale, est une énormité. Mais l'arrestation de Pinochet a provoqué un changement. Tous les sondages d'opinion montraient que $70 \%$ de la population pensait que Pinochet devait être jugé. Il y a donc une incohérence qu'on ne peut comprendre que dans le cadre de la transition démocratique du Chili, qui date de dix ans. Un processus difficile à comprendre, lié à notre mémoire historique et qui signifie le maintien des institutions de la dictature avec des réformes 
qui n'en ont jamais modifié l'essence. C'est ce qui est terrible... ce qui provoque la crise actuelle et que l'arrestation de Pinochet a mis en évidence.

19. Je crois que la force de la mémoire nous permettra de guérir. C'est pour ça qu'il faut établir la mémoire collective... pour pouvoir vivre et construire l'avenir.

20. Le lecteur trouvera dans une «note d'intention " inédite et que j'inclus en annexe à mon article plus d'explications à ce sujet. On peut également lire dans cette note une introduction biographique de la réalisatrice, une introduction historique du film ainsi qu'une explication des raisons qui l'ont poussée à le réaliser.

21. La classe populaire est représentée par la famille Arcos, la bourgeoisie par Mónica Salinas et ses amies, il y a aussi un avocat, Fernando Barros (fondateur d'un mouvement civique de soutien à Pinochet à Londres, où il résidait quand le dictateur fut détenu); une "artiste ", Patricia Maldonado, un capitaine de l'armée, Bernardo Acuña, Enrique Jara, candidat de l'UDI et représentant de la jeunesse du parti de la droite chilienne, Christian Labbé, maire de «Providencia » (un des quartiers les plus riches de Santiago, ancien colonel et secrétaire général du gouvernement militaire, qui occupa des responsabilités à la DINA, Service d'intelligence de la police politique pendant la dictature), Fernando Moreno Valencia, docteur en philosophie et un des mentors intellectuels de la dictature, Raúl Hasbún, représentant des secteurs les plus conservateurs de l'Église catholique, des éditorialistes de El Mercurio (journal conservateur chilien), des étudiants universitaires...

22. On pourrait souligner aussi les propos de Christian Labbé qui analyse l'intervention de l'armée comme une continuation logique et traditionnelle du rôle de l'armée au Chili. Or, comme l'explique très bien l'historien Alfredo Jocelyn Holt, bien que l'armée chilienne ait joué un rôle important dans la création de la nation depuis l'indépendance, la tradition chilienne est démocratique et ce rôle n'a jamais donné lieu à l'institutionnalisation de son pouvoir. Spécialement alarmants, puisqu'ils s'adressent à de futurs militaires, sont aussi les propos de Bernardo Acuña à ses élèves de l'École prémilitaire «tout ce qu'on a de bon, affirme-t-il, c'est le gouvernement militaire qui nous l'a laissé... ».

23. Mónica Salinas elle-même nous donne un exemple pathétique de cela : étant à Londres pour soutenir dans la rue Pinochet, elle se déguise en drapeau... plus tard à Santiago elle montre avec fierté à la caméra les photos avec Pinochet où elle apparaît ainsi vêtue...

24. On peut consulter une analyse complète des chiffres dans différents documents accessibles sur Internet (voir par exemple <http://www.unicef.cl/unicef/index.php/Pobreza>). Malgré la polémique sur les procédures d'évaluation de la pauvreté, en tout état de cause elle était beaucoup plus importante pendant la dictature et elle n'a cessé de diminuer depuis l'arrivée de la démocratie (même si les inégalités sociales restent encore un des problèmes majeurs à résoudre). 25. Pendant dix-sept ans l'image de Pinochet en train d'inaugurer des logements sociaux fut peut-être l'image la plus répétée de la télévision chilienne. La dictature ne manqua pas de propagande qui, jour après jour, essayait de nous convaincre de combien le gouvernement militaire était bénéfique et fructifiant.

26. En 1988 Pinochet convoque un plébiscite après seize ans de régime militaire. $44 \%$ de la population se prononce en sa faveur, $44 \%$ des Chiliens se déclarent ouvertement pro-Pinochet. En 1999 le candidat de la coalition de droite, Joaquin Lavin, qui, en 1988 incite les Chiliens à voter pour Augusto Pinochet, obtient $47 \%$ des votes. Il est préoccupant de constater qu'un héritier direct de la dictature s'annonce comme le futur président du Chili, il est plus inquiétant encore de constater que tous les secteurs de la société, sans distinction, soient perméables à ses opinions.

27. Cette même nécessité d'un Chili pour tous reste encore d'actualité quelques années plus tard. C'est cela qu'affirme Claire d'Hennezel lorsqu'elle analyse la première année du gouvernement de M. Bachelet : «L'assainissement en profondeur des pratiques politiques chiliennes est sans aucun doute le projet le plus important et le plus significatif du gouvernement de Michelle Bachelet. Il 
porte en lui la promesse d'une mutation à long terme du pays. De colosse économique érigé sous une chape de plomb dans les années 1980, conciliant tant bien que mal réussite économique et sociale dans les années 1990, le pays s'apprête, en 2007, à relever avec succès un nouveau défi : celui de devenir le Chili de tous les Chiliens. La lutte contre toutes les formes d'exclusion devra, dans les années à venir, combler les déficits actuels de démocratie du modèle de développement chilien. » 28. La victoire de Michelle Bachelet en mars 2006 a été analysée d'ailleurs comme preuve irréfutable de cet aboutissement. Comme l'exprime Emmanuelle Barozet, Bachelet « incarne une certaine forme de réconciliation nationale » (2007, p. 22-27). Parallèlement à la victoire de Bachelet, les procès judiciaires ouverts contre Pinochet, mis en examen et assigné à résidence, la discréditation ensuite de la famille Pinochet, impliquée dans des affaires de corruption, de détournement de fonds publics et d'évasion fiscale, ainsi que la mort du vieil homme déchu qu'était devenu Pinochet, signifient une sorte de double victoire symbolique. Pour la sociologue chilienne, qui intitule son article « Après la transition, quelle démocratie ? » suite à la victoire de Bachelet, la transition serait une période effectivement aboutie. Il ne serait plus question désormais que de savoir quelle démocratie les Chiliens veulent construire. Nonobstant les difficultés que Bachelet a rencontrées pour effectuer les reformes nécessaires, la constitution encore aujourd'hui inamovible ainsi que la plus que possible victoire d'une droite très conservatrice aux prochaines élections, rendent compte de la pertinence, toujours valable, des réflexions du documentaire de Marcela Said.

29. «Je crois qu'en général les choses progressent lentement grâce au fait que d'importants secteurs de la société travaillent les problèmes de la mémoire et que l'intention d'occulter complètement tout ce qui arriva pendant la dictature n'a pas abouti (malgré les efforts de la droite et de la concertation) et que, avec le temps, les jeunes continueront à chercher les informations qu'ils n'ont jamais eues et à ce moment-là les documentaires seront une "mémoire vivante".»

\section{RÉSUMÉS}

L'arrestation d'Augusto Pinochet à Londres en 1998 marquera un point d'inflexion capital dans l'évolution de la démocratie chilienne. Entre autres, l'expression dans l'espace public des différentes réactions à cette arrestation témoignait d'une société chilienne divisée qui, progressivement, brisait la «loi du silence». Le consensus longtemps véhiculé par une démocratie, qui avançait tant bien que mal dans la continuité de certaines règles du jeu imposées par les militaires, semblait ainsi se dissiper.

Patricio Guzmán et Marcela Said vont s'emparer dans El caso Pinochet et I love Pinochet, films documentaires apparus en 2001, des voix de ces deux Chili confrontés dans la rue pour mieux scruter la portée de ce tournant historique. L'objectif de cet article est d'analyser ces films en tant que documents pour l'Histoire dans la mesure où ils apportent à la «lecture » des faits un regard critique qui se constitue en véritable revendication d'une nouvelle démocratie pour leur pays.

Augusto Pinochet's arrest in London in 1998 was a turning point in the evolution of Chilean democracy. The different publicly expressed reactions to his arrest revealed a divided society that was increasingly breaking the "law of silence". Indeed, the consensus long conveyed in this hardly surviving democracy, that continued to apply some of the rules imposed by the military 
government, seemed to be slowly vanishing.

In their documentary films released in 2001, El caso Pinochet and I love Pinochet, Patricio Guzman and Marcela Said captured the voice of those two Chili confronting each other in the streets, in order to better understand the impact of this historical turning point. The purpose of this paper is to analyze these films as documents for History as they take a critical look at the "reading" of historical facts, a look that becomes henceforth a real claim for a new democracy in their country.

\section{INDEX}

Keywords : Chili, democratic transition, documentary films, History and Memory, Pinochet case Mots-clés : Affaire Pinochet, Chili, cinéma documentaire, histoire et mémoire, transition démocratique

\section{AUTEUR}

\section{OLGA LOBO}

Docteure en études ibériques et ibéro-américaines, Centre d'études et de recherches hispaniques (CERHIUS) au sein de l'Institut des langues et des cultures d'Europe et d'Amérique (ILCEA), université Stendhal-Grenoble 3 